\title{
The role of the gut microbiota in acute kidney injury: a new therapeutic candidate?
}

\author{
Chan Ho Kim, Sung Jin Moon \\ Department of Internal Medicine, International St. Mary's Hospital, Catholic Kwandong University, Incheon, Republic of Korea
}

\section{See Article on Page 633-646}

The intestinal microbiota, a community of 100 trillion microorganisms (more than 1,000 species that consist of mostly bacteria but also viruses, fungi, and protozoa), plays an important role in maintaining homeostasis (especially in regard to mucosal immunity and nutrient metabolism) in the human gastrointestinal tract [1]. The intestinal epithelial barrier can be divided into three components; a biological barrier, a physical barrier, and an immune barrier [2]. The biological barrier is composed of bacterial and fungal symbionts that are closely attached to the intestinal mucosal surface and compete with pathogenic bacteria. The physical barrier refers to intestinal epithelial cells with apical tight junctions (TJs). Changes in TJs can lead to increased permeability, allowing bacteria, endotoxins, and macromolecules to enter the circulatory system. The immune barrier is the third system for maintaining microbial homeostasis. Dendritic cells in the lamina propria activate $\mathrm{T}$ cells to evoke an adaptive immune response. Innate lymphoid cells located in the gut epithelium have key defensive functions to produce or activate the release of immune-activating cytokines.

Recent studies suggest that altered structure and composition of gut microbiota known as dysbiosis are linked to disrupted homeostasis and pathogenesis of various diseases, including inflammatory bowel disease, obesity, diabetes, cancer, Alzheimer disease, nonalcoholic fatty liver disease, and chronic obstructive pulmonary disease [1-3]. Therefore, several hypotheses of the "Gut-Brain Axis," "Gut-Liver Axis," "Gut-Lung Axis," and others have been proposed to explain the bidirectional complex communication between the gut and other organs. Although the exact mechanism underlying crosstalk between gut microbiota and distant organs remains uncertain, it has been proposed that the pathogenesis might be mediated by altering the function of the intestinal barrier, modifying local and systemic inflammation, controlling the production of metabolites, and affecting immune responses $[1,3,4]$.

From the point of view of kidney disease, several recent experimental and clinical data have revealed the existence of a gut-kidney axis and the pivotal role of gut microbiota in kidney injury $[3,5]$. Increasing urea concentration in chronic kidney disease (CKD) leads to alterations in the intestinal flora. The potential mechanisms for change in

Received: October 24, 2021; Accepted: October 24, 2021

Editor: Tae-Hyun Yoo, Yonsei University, Seoul, Republic of Korea

Correspondence: Sung Jin Moon

Department of Internal Medicine, International St. Mary's Hospital, Catholic Kwandong University, 25 Simgok-ro 100beon-gil, Seo-gu, Incheon 22711, Republic of Korea. E-mail: moonsj75@ish.ac.kr

ORCID: https://orcid.org/0000-0002-6276-3009

Copyright (@ 2021 by The Korean Society of Nephrology

(a) This is an Open Access article distributed under the terms of the Creative Commons Attribution Non-Commercial and No Derivatives License (http:// creativecommons.org/licenses/by-nc-nd/4.0/) which permits unrestricted non-commercial use, distribution of the material without any modifications, and reproduction in any medium, provided the original works properly cited. 
microbiome composition include decreased fiber intake, phosphate binder use, decreased colonic transit time, and comorbidities such as diabetes. Alterations in the intestinal flora can increase the production of gut-derived toxins and alter the intestinal epithelial barrier. These changes can accelerate the process of kidney injury. Probiotics are "live microorganisms that, when administered in adequate amounts, confer a health benefit on the host" and have been used as a potential therapeutic option in several chronic inflammatory disease models, including CKD [6].

To date, only a limited number of studies have investigated the impact of probiotics in the gut-kidney axis during acute kidney injury (AKI). Yang et al. [7] investigated the beneficial effects and distant organ influences of prior probiotic supplementation on AKI in a renal ischemia/reperfusion injury (IRI) animal model. Pretreatment of mice with Bifidobacterium bifidum BGN4 (BGN4) attenuated AKI-induced dysbiosis and gut barrier disruption. In addition, BGN4 administration significantly reduced the severity of renal IRI and distant organ (liver) injury. These results were associated with expansion of regulatory $\mathrm{T}$ cells and reduced interleukin-17A expression in the colon, mesenteric lymph nodes, and kidney, suggesting that BGN4-induced immunomodulation contributes to its renoprotective effect. Previously, the authors demonstrated a bidirectional relationship between the kidney and intestine in AKI that renal IRI provoked intestinal dysbiosis and the dysbiotic microbiota was an important modifier of postischemic kidney outcome due to immune modulatory effects [8]. Moreover, they also reported that Lactobacillus salivarius BP121 attenuated cisplatin-induced kidney injury by decreasing inflammation, oxidative stress, and serum levels of uremic toxins and by modulating the gut environment [9]. Recently, some studies have presented the renoprotective effects of probiotics and gut microbiota-derived metabolites (e.g., short-chain fatty acids [SCFAs] and D-serine). Lactobacillus casei Zhang delayed kidney disease progression in a renal IRI mouse model and also in patients with CKD by increasing the levels of SCFAs and via nicotinamide metabolism, which together modulate the inflammatory response of local macrophages and tubular epithelial cells [6]. Furthermore, administration of SCFAs (acetate, propionate, and butyrate) attenuated inflammation in kidney epithelial and immune cells and ameliorated renal IRI through modula-

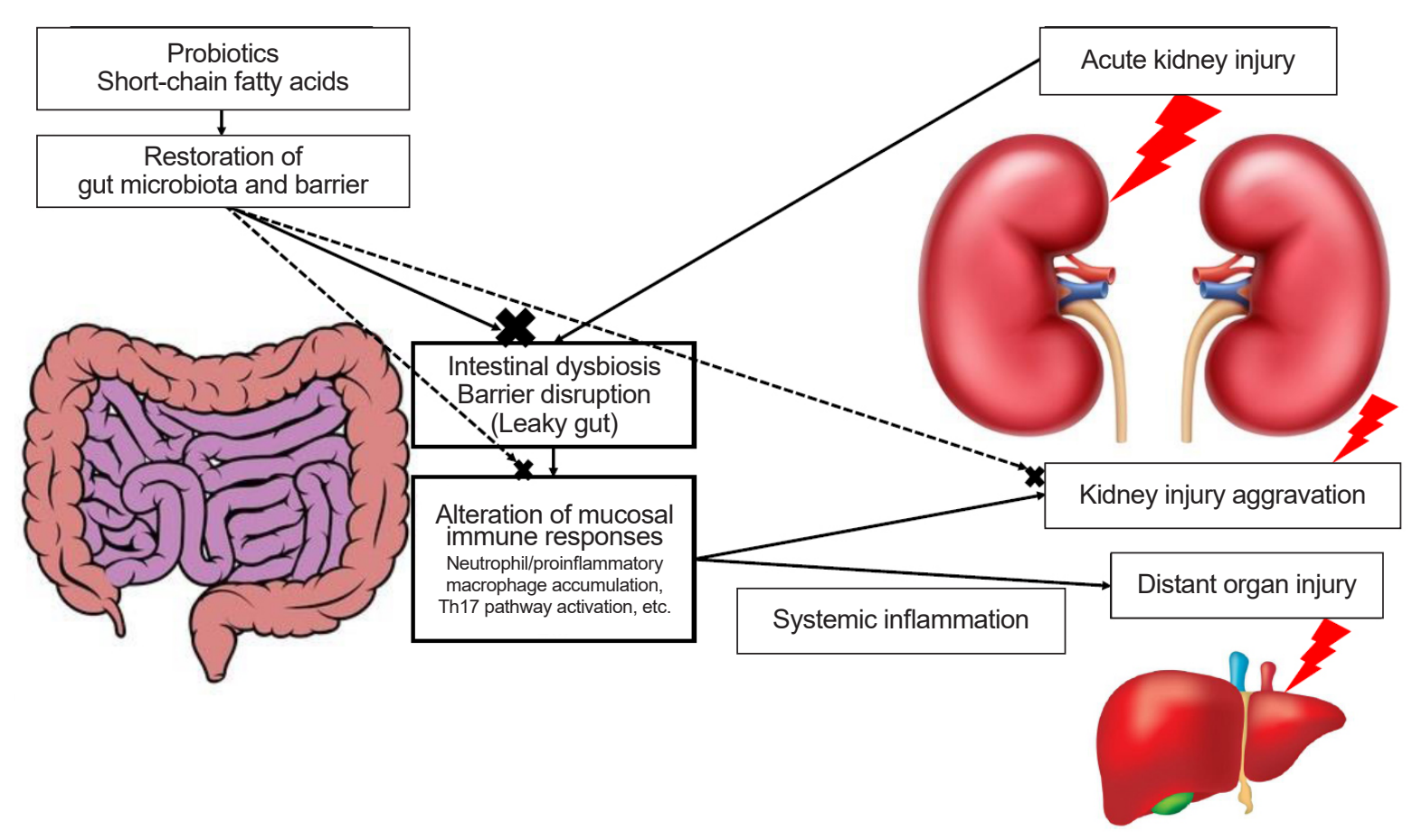

Figure 1. The bidirectional relationship between the gut microbiota and kidney and the proposed protective role of probiotics and their metabolites in acute kidney injury. 
tion of the inflammatory process, most likely via epigenetic modification. Gut microbiota-derived D-serine was shown to reduce tubular damage in AKI. In addition, a microbial cocktail of Escherichia, Bacillus, and Enterobacter exhibited a protective effect in nephrotoxin-induced AKI [10]. Although these studies indicate the bidirectional relationship between the intestinal microbiota and kidney through the gut-kidney axis in AKI, the precise mechanisms by which these processes act remain unclear.

Based on the studies mentioned above, crosstalk in the gut-kidney axis during AKI can be explained as follows. (1) AKI induces intestinal dysbiosis and barrier disruption (often referred to as "leaky gut"); (2) dysbiosis and leaky gut alter mucosal immune responses, which leads to accumulation of neutrophils and proinflammatory macrophages and activation of the Th17 pathway; (3) a shift in mucosal immunity toward proinflammatory status can aggravate kidney and distant organ injury via systemic inflammation; and (4) probiotics or their metabolites might have a potential renoprotective effect by restoring the intestinal microbiota and gut environment through immune modulation (Fig. 1).

In summary, the interactions between the gut microbiota and the kidney are important in AKI, and probiotics are a potential therapeutic approach for AKI (Fig. 1). However, the research results were insufficient because they have not been applied to humans, and it remains unclear whether administration of probiotics after AKI development has a protective effect on renal function. Future studies are needed to clarify the role of the intestinal microbiota in AKI and to explore whether modification of the gut microbiota using probiotics or supplementation with their metabolites is a potential therapeutic option.

\section{Conflicts of interest}

All authors have no conflicts of interest to declare.

\section{ORCID}

Chan Ho Kim, https://orcid.org/0000-0001-5104-5194

Sung Jin Moon, https://orcid.org/0000-0002-6276-3009

\section{References}

1. Fung TC, Olson CA, Hsiao EY. Interactions between the microbiota, immune and nervous systems in health and disease. Nat Neurosci 2017;20:145-155.

2. Gong J, Noel S, Pluznick JL, Hamad AR, Rabb H. Gut microbiota-kidney cross-talk in acute kidney injury. Semin Nephrol 2019;39:107-116.

3. Noel S, Martina-Lingua MN, Bandapalle S, et al. Intestinal microbiota-kidney cross talk in acute kidney injury and chronic kidney disease. Nephron Clin Pract 2014;127:139-143.

4. Al Khodor S, Shatat IF. Gut microbiome and kidney disease: a bidirectional relationship. Pediatr Nephrol 2017;32:921-931.

5. Rydzewska-Rosołowska A, Sroka N, Kakareko K, Rosołowski M, Zbroch E, Hryszko T. The links between microbiome and uremic toxins in acute kidney injury: beyond gut feeling: a systematic review. Toxins (Basel) 2020;12:788.

6. Zhu H, Cao C, Wu Z, et al. The probiotic L. casei Zhang slows the progression of acute and chronic kidney disease. Cell Metab 2021;33:1926-1942.

7. Yang J, Ji GE, Park MS, Seong YJ, et al. Probiotics partially attenuate the severity of acute kidney injury through an immunomodulatory effect. Kidney Res Clin Pract 2021;40:633-646.

8. Yang J, Kim CJ, Go YS, et al. Intestinal microbiota control acute kidney injury severity by immune modulation. Kidney Int 2020;98:932-946.

9. Lee TH, Park D, Kim YJ, et al. Lactobacillus salivarius BP121 prevents cisplatin-induced acute kidney injury by inhibition of uremic toxins such as indoxyl sulfate and p-cresol sulfate via alleviating dysbiosis. Int J Mol Med 2020;45:1130-1140.

10. Zheng DW, Pan P, Chen KW, et al. An orally delivered microbial cocktail for the removal of nitrogenous metabolic waste in animal models of kidney failure. Nat Biomed Eng 2020;4:853-862. 